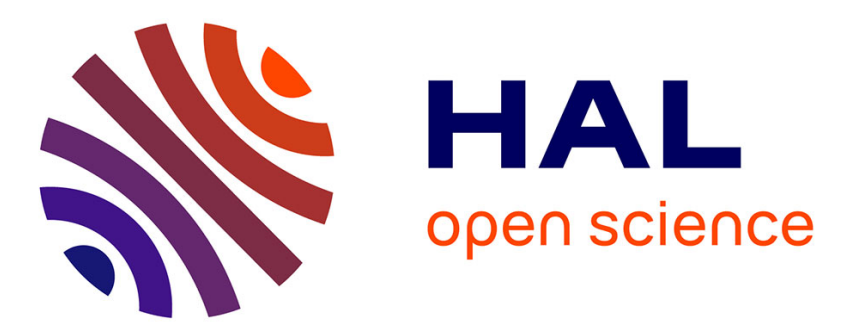

\title{
Some Co-Axial Magnetic Couplings Designed Using an Analytical Model and an Exact Global Optimization Code
}

Julien Fontchastagner, Yvan Lefèvre, Frédéric Messine

\section{- To cite this version:}

Julien Fontchastagner, Yvan Lefèvre, Frédéric Messine. Some Co-Axial Magnetic Couplings Designed Using an Analytical Model and an Exact Global Optimization Code. IEEE Transactions on Magnetics, 2009, 45 (3), pp.1458-1461. 10.1109/TMAG.2009.2012678 . hal-01522924

\section{HAL Id: hal-01522924 \\ https://hal.science/hal-01522924}

Submitted on 15 May 2017

HAL is a multi-disciplinary open access archive for the deposit and dissemination of scientific research documents, whether they are published or not. The documents may come from teaching and research institutions in France or abroad, or from public or private research centers.
L'archive ouverte pluridisciplinaire HAL, est destinée au dépôt et à la diffusion de documents scientifiques de niveau recherche, publiés ou non, émanant des établissements d'enseignement et de recherche français ou étrangers, des laboratoires publics ou privés. 


\title{
Some Co-Axial Magnetic Couplings Designed Using an Analytical Model and an Exact Global Optimization Code
}

\author{
Julien Fontchastagner ${ }^{1}$, Yvan Lefèvre ${ }^{1}$, and Frédéric Messine ${ }^{2}$ \\ ${ }^{1}$ Laboratoire Plasma et Conversion d'Energie (LAPLACE), Université de Toulouse-CNRS, BP 7122, 31071 INPT-ENSEEIHT, \\ Toulouse Cedex 7, France \\ ${ }^{2}$ Institut de Recherche en Informatique de Toulouse (IRIT), Université de Toulouse-CNRS, BP 7122, 31071 INPT-ENSEEIHT, \\ Toulouse Cedex 7, France
}

\begin{abstract}
The purpose of this paper is to use a new rational approach for the design of some magnetic couplings. Our method is based on the association of analytical models and an exact global optimization algorithm named IBBA and developed by the third author. The analytical model presented in this paper is more sophisticated than those previously dealt using IBBA. Therefore, some under and over estimating functions have been constructed for those particular problems of design in order to improve the convergence of IBBA. Some optimal results highlight the efficiency of this approach.
\end{abstract}

Index Terms-Analytical model, Branch\&Bound, exact global optimization, interval arithmetic, inverse problem, magnetic coupling.

\section{INTRODUCTION}

$\mathbf{T}$ HE problem of design is here understood and formulated as an inverse problem. This methodology is well adapted to the design of some electromechanical actuators, see [1], [2]. In order to solve exactly the so-generated global optimization problems, a particular deterministic global algorithm based on a branch and bound technique and on interval arithmetic has been developed by the third author, [3]. In [1] and [2], IBBA was associated with analytical models of permanent magnets motors to find optimized solutions of design.

The associated analytical models need several assumptions to get simplified expressions of electromechanical quantities such as the flux and the torque. Therefore, when optimal solutions of a design problem are obtained, it is often necessary to validate them by means of a numerical model. In order to reduce the use of a numerical model, an analytical model that needs fewer assumptions is proposed. This model is based on the method of separation of the variables for solving Poisson's equations. It gives results that are very close to those obtained by using a finite-element analysis (FEA), [4], [5].

In this paper, IBBA is used once more time but applied to a new application of design concerning magnetic couplings. That is the first time that IBBA is associated with such complicated analytical expressions and thus, some ideas for constructing over and under estimating functions have been introduced. Due to the particular geometrical structure of such devices, it is possible to solve Maxwell equations by separation of the variables. The analytical expressions of electromechanical quantities are more complicated than those employed in [2] but are far simpler than the ones in [4] and [5]. In our knowledge, no other optimization method has been used to solve exactly those kinds of design problems.

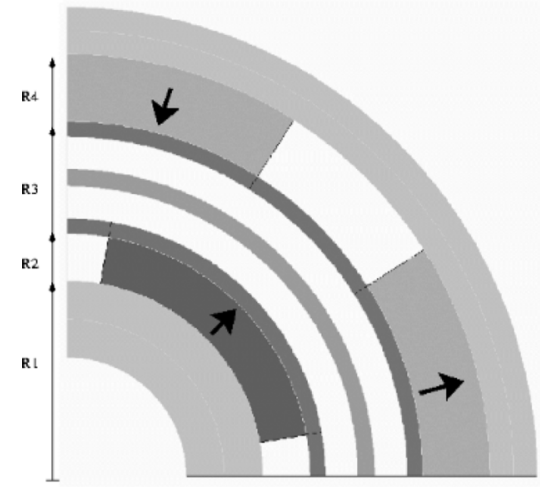

Fig. 1. Geometry of the considered magnetic coupling.

The analytical model is presented in Section II. In Section III, the principle of IBBA is summarized. Its adaptation to our problem is detailed. Section IV presents some magnetic couplings which are optimized using this methodology.

\section{MAGNETIC COUPLING}

Magnetic coupling are very useful devices for many applications as in chemical and petrochemical industries. In an effort to prevent leakage of hazardous fluids from piping systems, the use of magnetically driven sealless pumps has become more common. Magnetic couplings are used in them to transmit rotational motion without mechanical contact. The studied structure is a co-axial magnetic coupling as shown on Fig. 1. It consists of two rings of permanent magnets separated by a containment can. On each side of it, we find an airgap and a binding band.

\section{A. Parameterization}

In order to calculate the torque produced by such a device, one must first choose the parameters required for the computation of the magnetic fields inside it. On Fig. 1, we can see that the two rings of magnet are separated by five concentric areas: two binding bands, two airgap zones and, in the middle, the containment can. These zones are all nonmagnetic, thus we include them into only one big area named airgap thereafter. Moreover, we can consider that the two rings of iron yoke have an infinite permeability. Thus, we can take into account only three areas: Area $^{1}$ is the inner ring of magnets, Area $^{3}$ the outer one and 
TABLE I

DEFINITION OF PARAMETERS

\begin{tabular}{|c|c|c|}
\hline Sy mbol & Quant iy & Un its \\
\hline int & Angul ar length of an inter ior magnet & $\mathrm{rad}$ \\
\hline$e x t$ & Angular length of an ext er ior magnet & $\mathrm{rad}$ \\
\hline$R_{l}$ & Inner radius of int er ior magnet s & $\mathrm{m}$ \\
\hline$R_{2}$ & Out er radius of int er ior magnet $\mathrm{s}$ & $\mathrm{m}$ \\
\hline$R_{3}$ & Inner radius of ext er ior magnet $\mathrm{m}$ & $\mathrm{m}$ \\
\hline$R_{4}$ & Out er radius of exter ior magnet s & $\mathrm{m}$ \\
\hline$L$ & Common length of the act ive magnet ic parts & $\mathrm{m}$ \\
\hline$p$ & Number of pole pa ir s $(\geq 2)$ & - \\
\hline$\sigma_{\mathrm{mi}}$ & Kind of inter ior magnet & - \\
\hline$\sigma_{\mathrm{me}}$ & Kind of exter ior magnet & - \\
\hline$\sigma_{\mathrm{vi}}$ & Kind of inner iron yoke mater ial & - \\
\hline$\sigma_{\mathrm{ve}}$ & Kind of out er iron yoke mat er ial & - \\
\hline
\end{tabular}

Area $^{2}$ the airgap. Our structure can be described by 12 parameters ( 7 reals, 1 integer, 4 categorical variables, see Table I).

\section{B. Modeling}

In [4] and [5], an analytical model of the magnetic field in permanent magnet motors is proposed. In [5], a magnetic scalar potential formulation is used. We find the magnetic vector potential formulation described in [4] suitable for magnetic couplings.

1) Magnetic Vector Potential Formulation: As the two armatures of a magnetic coupling rotate at the same speed, the magnetic field can be considered static in a reference fixed on one of them and static Maxwell equations may be applied [4]. The constitutive laws of magnetic media are as follows:

— in the airgap $\left(\right.$ Area $\left.^{2}\right)$

$$
\mathbf{B}=\mu_{0} \mathbf{H} .
$$

— In the permanent magnets (Area ${ }^{1}$ and Area $^{3}$ )

$$
\mathbf{B}=\mu_{0} \mathbf{H}+\mathbf{J} .
$$

From the Gauss's law of magnetism, we can derive the magnetic field from a magnetic vector potential, A. Owing to the geometry of the magnetic coupling (see Fig. 1), we can assume a translational invariance along the $\mathrm{Oz}$ axis. Thus, the vector potential has only one non-null component: $A_{z}$. The magnetic polarizations of magnets $\mathbf{J}$ are assumed to be purely radial. They have a spatial period equal to $2 \pi / p$ in the $\theta$ direction. Therefore, in polar coordinates, we can write the harmonic series [4]

$$
J_{r}(r, \theta)=\sum_{n=1}^{\infty} J_{\mathrm{rcn}} \cos (n p \theta)+J_{\mathrm{rsn}} \sin (n p \theta) .
$$

The partial differential equation governing $A_{z}$ is [4]

$$
\frac{\partial^{2} A_{z}}{\partial r^{2}}+\frac{1}{r} \frac{\partial A_{z}}{\partial r}+\frac{1}{r^{2}} \frac{\partial^{2} A_{z}}{\partial \theta^{2}}= \begin{cases}0, & \text { in Area } \\ \frac{1}{r} \frac{\partial J_{r}}{\partial \theta}, & \text { otherwise. }\end{cases}
$$

To these equations, we must add the boundary conditions and the continuity conditions between two media. As the inner and outer yokes are assumed to have an infinite permeability, we have on surface boundaries at $\left(r=R_{1}\right)$ and $\left(r=R_{4}\right)$ :

$$
\left(\frac{\partial A_{z}^{1}}{\partial r}\right)_{r=R_{1}}=0, \quad\left(\frac{\partial A_{z}^{3}}{\partial r}\right)_{r=R_{4}}=0
$$

where $A_{z}^{i}$ is the value of $A_{z}$ in a media $\operatorname{Area}^{i}, A_{z}^{i}$. Thus, the continuity conditions of the magnetic field must be applied on the interfaces at $\left(r=R_{2}\right)$ and $\left(r=R_{3}\right)$. For instance, on interfaces $\left(r=R_{2}\right)$, we have

$$
\left(\frac{\partial A_{z}^{1}}{\partial \theta}\right)_{r=R_{2}}=\left(\frac{\partial A_{z}^{2}}{\partial \theta}\right)_{r=R 2},\left(\frac{\partial A_{z}^{1}}{\partial r}\right)_{r=R_{2}}=\left(\frac{\partial A_{z}^{2}}{\partial r}\right)_{\substack{r=R_{2} \\(6)}} .
$$

Because $(p \geq 2)$, the method of separation of the variables gives the form of the analytical expression of $A_{z}$ in Area $^{i}$ :

$$
\begin{aligned}
A_{z}^{i}(r, \theta) & =\sum_{n=1}^{\infty}\left[\left(A_{n}^{i} r^{n p}+B_{n}^{i} r^{-n p}+\frac{s c_{n}^{i} r}{1-(n p)^{2}}\right) \cos (n p \theta)\right. \\
+ & \left.\left(C_{n}^{i} r^{n p}+D_{n}^{i} r^{-n p}+\frac{s s_{n}^{i} r}{1-(n p)^{2}}\right) \sin (n p \theta)\right] .
\end{aligned}
$$

The $s c_{n}^{i}$ and $s s_{n}^{i}$ coefficients are null in Area $^{2}$. In Area ${ }^{1}$ and Area $^{3}$, we have the following relations:

$$
\begin{aligned}
& s c_{n}^{1}=0 \\
& s s_{n}^{1}=-\frac{2 p J_{r}\left(\sigma_{\text {int }}\right)}{\pi} \sin \left(\frac{n p \theta_{\text {int }}}{2}\right)\left(1-(-1)^{n}\right) \\
& s c_{n}^{3}=-\frac{p J_{r}\left(\sigma_{\text {ext }}\right)}{\pi}\left[\cos \left(\frac{n p \theta_{\text {ext }}}{2}+n p \theta_{0}\right)\right. \\
&\left.-\cos \left(-\frac{n p \theta_{\text {ext }}}{2}+n p \theta_{0}\right)\right]\left(1-(-1)^{n}\right) \\
& s s_{n}^{3}=-\frac{p J_{r}\left(\sigma_{\text {ext }}\right)}{\pi}\left[\sin \left(\frac{n p \theta_{\text {ext }}}{2}+n p \theta_{0}\right)\right. \\
&\left.\quad-\sin \left(-\frac{n p \theta_{\text {ext }}}{2}+n p \theta_{0}\right)\right]\left(1-(-1)^{n}\right)
\end{aligned}
$$

where $\theta_{0}$ is the angular gap between the two armatures and $J_{r}\left(\sigma_{\text {int }}\right)$ and $J_{r}\left(\sigma_{\text {ext }}\right)$ are respectively the radial polarization of inner and outer magnets. The value of the torque is maximum when $\theta_{0}=\pi / 2$ (see Fig. 1). Thanks to relations (5) and (6) the values of $A_{n}^{i}, B_{n}^{i}, C_{n}^{i}$, and $D_{n}^{i}$ coefficients appearing in relation (7) can be known by solving the two equations

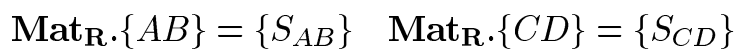

where

$$
\begin{aligned}
& \{A B\}^{T}=\left\{A_{n}^{1}, B_{n}^{1}, A_{n}^{2}, B_{n}^{2}, A_{n}^{3}, B_{n}^{3}\right\} \\
& \{C D\}^{T}=\left\{C_{n}^{1}, D_{n}^{1}, C_{n}^{2}, D_{n}^{2}, C_{n}^{3}, D_{n}^{3}\right\} \\
& \left\{S_{A B}\right\}^{T}=k_{n p}\left\{0,0,0, n p R_{3} s c_{n}^{3}, R_{3} s c_{n}^{3}, R_{4} s c_{n}^{3}\right\} \\
& \left\{S_{C D}\right\}^{T}=k_{n p}\left\{R_{1} s s_{n}^{1}, n p R_{2} s s_{n}^{1}, R_{2} s s_{n}^{1}, n p R_{3} s s_{n}^{3},\right. \\
& k_{n p}=\frac{1}{n p\left(1-(n p)^{2}\right)}
\end{aligned}
$$

$\operatorname{Mat}_{\mathbf{R}}=$

$$
\left(\begin{array}{cccccc}
-R_{1}^{n p} & R_{1}^{-n p} & 0 & 0 & 0 & 0 \\
-R_{2}^{n p} & -R_{2}^{-n p} & R_{2}^{n p} & R_{2}^{-n p} & 0 & 0 \\
-R_{2}^{n p} & R_{2}^{-n p} & R_{2}^{n p} & -R_{2}^{-n p} & 0 & 0 \\
0 & 0 & R_{3}^{n p} & R_{3}^{-n p} & -R_{3}^{n p} & -R_{3}^{-n p} \\
0 & 0 & R_{3}^{n p} & -R_{3}^{-n p} & -R_{3}^{n p} & R_{3}^{-n p} \\
0 & 0 & 0 & 0 & -R_{4}^{n p} & R_{4}^{-n p}
\end{array}\right) .
$$

2) Magnetic Torque: The magnetic flux density $\mathbf{B}^{\mathbf{i}}$ in each Area $^{i}$ can be calculated from the magnetic vector potential $A z$. 
Hence, by applying the Maxwell stress tensor in the airgap, the torque $\Gamma_{\text {em }}$ can be obtained. Comparisons with FEA show that we can take into account only the first harmonic $(n=1)$. Thus, using MAPLE, we can get an analytical expression of the torque (10)

$$
\begin{aligned}
\Gamma_{\mathrm{em}}= & \frac{-8 p L}{\mu_{0} \pi\left(p^{2}-1\right)^{2}\left(R_{4}^{2 p}-R_{1}^{2 p}\right)} \\
& \times J_{r}\left(\sigma_{m i}\right) \sin \left(p \theta_{\mathrm{int}} / 2\right) J_{r}\left(\sigma_{m e}\right) \sin \left(p \theta_{\mathrm{ext}} / 2\right) \\
& \times\left((p-1) R_{2}^{p+1}+2 R_{1}^{p+1}-(p+1) R_{1}^{2 p} / R_{2}^{p-1}\right) \\
& \times\left((p-1) R_{3}^{p+1}+2 R_{4}^{p+1}-(p+1) R_{4}^{2 p} / R_{3}^{p-1}\right) .
\end{aligned}
$$

3) Thickness of Iron Yokes: We present here a simple method which allows to calculate an approximation of the thickness of each iron yoke $\left(t_{y}\right)$. Indeed, the mean value of magnetic flux density $B_{\theta}^{y}$ in the yokes must be less or equal to the maximum value $B_{M}\left(\sigma_{y}\right)$ above which the iron is definitely saturated.

In the yoke, the maximum value of magnetic flux is $\Phi_{y}=$ $B_{\theta}^{y} t_{y} L$ and the magnetic flux per pole is given by

$$
\Phi_{m}=\iint \mathbf{B} \cdot \mathbf{d} \mathbf{S}=\int_{\theta}\left(\frac{1}{r} \frac{\partial A_{z}}{\partial \theta}\right) \operatorname{Lr} d \theta=2 L \cdot \operatorname{Max}\left(A_{z}\right)
$$

where $\operatorname{Max}(A z)$ is the maximum value of the magnetic vector potential and can be obtained from relations (7) and (9). According to the Gauss's law of magnetism we have $\Phi_{m}=2 \Phi_{y}$. Eventually, the analytical expression of the thickness of the inner yoke $t_{y}^{1}$ is

$$
\begin{aligned}
t_{y}^{1}= & \frac{4 J_{r}\left(\sigma_{m i}\right) \sin \left(p \theta_{\mathrm{int}} / 2\right)}{\pi B_{M}\left(\sigma_{y i}\right)\left(p^{2}-1\right)\left(R_{4}^{2 p}-R_{1}^{2 p}\right)}\left[(p-1) R_{1}^{2 p}\right. \\
& \left.\left(R_{2}^{p+1}-R_{1}^{p+1}\right)+(p+1) R_{4}^{2 p} R_{1}\left(1-\left(\frac{R_{1}}{R_{2}}\right)^{p-1}\right)\right]
\end{aligned}
$$

where $B_{M}\left(\sigma_{y i}\right)$ is the maximum flux density in the inner iron yoke. An analogue expression for the thickness of the outer yoke $t_{y}^{3}$ can be derived.

\section{Formalization of THE DESIGN PROBlem}

\section{A. Formulation}

An inverse problem is formulated as mixed constrained global optimization problems defined as follows:

$$
\left\{\begin{array}{l}
\min _{\substack{x \in R^{n}, z \in N^{m} \\
\sigma \in \prod_{i=1}^{l} K^{i}, b \in B^{r}}} f(x, z, \sigma, b) \\
\text { subjected to: } \\
\quad g_{i}(x, z, \sigma, b) \leq 0 \forall i \in\{1, \ldots, p\} \\
\quad h_{j}(x, z, \sigma, b)=0 \forall j \in\{1, \ldots, q\}
\end{array}\right.
$$

where $f$ is a real function; $K^{i}$ represents an enumerated set of categorical variables, that is for example the type of magnet; and $B=\{0,1\}$ the boolean set which is used to model, for example, the fact that an actuator is with or without slot(s).

\section{B. IBBA Algorithm}

Interval analysis was introduced by Moore in 1966 [6]. All real values are enclosed by an interval where the bounds
TABLE II

LIST OF CONSTRAINTS

\begin{tabular}{|c|c|}
\hline Number & Relation \\
\hline$C_{l}$ & $\Gamma_{e m}=\Gamma \mathrm{a}_{\text {fixed }}$ \\
\hline$C_{2}$ & $R_{2}-R_{1} \geq t_{y i}{ }^{\text {min }}$ \\
\hline$C_{3}$ & $R_{3}-R_{2} \geq g^{\text {min }}$ \\
\hline$C_{4}$ & $R_{3}-R_{2} \leq g^{\text {max }}$ \\
\hline$C_{5}$ & $R_{4}-R_{3} \geq t_{v e}{ }^{\text {min }}$ \\
\hline$C_{6}$ & $R_{4} \geq \lambda^{l} L$ \\
\hline$C_{7}$ & $R_{4} \leq \lambda^{u} L$ \\
\hline$C_{8}$ & $R_{1}-t_{v}{ }^{l} \geq R_{\text {int }}{ }^{\text {min }}$ \\
\hline
\end{tabular}

are the two closest floating point numbers. Expanding the classical operations-addition, subtraction, multiplication and division-into intervals, defines interval arithmetic. A straightforward generalization allows computations of reliable bounds of a function over a hypercube (or box) defined by an interval vector. Moreover, classical tools of analysis such as Taylor expansions can be used together with interval arithmetic to compute more precise bounds [6], [7].

The principle of IBBA is first to bisect the initial domain into smaller and smaller boxes and then, to eliminate the boxes where the global optimum cannot occurs:

- by proving, using interval bounds, that no point in a box can produce a better solution than the current best one;

- by proving, with interval arithmetic, that at least one constraint cannot be satisfied by any point in such a box.

To accelerate the convergence, constraint propagation techniques are used in some steps of IBBA [8]. Such interval Branch and Bound algorithms guarantee to produce an $\varepsilon$-global optimal solution, where $\varepsilon>0$ is the maximal error on the objective function value; $\varepsilon$ is fixed by the user of IBBA. Actually, IBBA was used to solved exactly some non-homogeneous mixed constrained global optimization problem with almost 25 variables and 15 parameters (taking between few seconds to 6 days of computing times). The difficulty to solve a problem depends on the number of variables and of constraints but also on the complexity of the analytical equations. Indeed interval arithmetic has not all the properties of the classical one (for example it is sub-distributive). Therefore, bounds computed using interval arithmetic can be very large [6]. This is the main difficulty that we have to deal with in this work. For details on such an algorithm see [3] and [7].

\section{Application and Adaptations of IBBA}

Our input vector is defined by the parameters used in Table I. The minimum value of the volume of magnets $\mathbf{V}_{\mathbf{a}}$ or the global volume $\mathbf{V}_{\mathbf{g}}$ is searched

$$
\begin{aligned}
& \mathbf{V}_{\mathbf{a}}=p L\left(\theta_{\text {int }}\left(R_{2}^{2}-R_{1}^{2}\right)+\theta_{\text {ext }}\left(R_{4}^{2}-R_{3}^{2}\right)\right. \\
& \mathbf{V}_{\mathbf{g}}=p L\left[\left(R_{4}+t_{y}^{3}\right)^{2}-\left(R_{1}-t_{y}^{1}\right)^{2}\right]
\end{aligned}
$$

Each solution must satisfy an equality constraint upon the fixed electromagnetic torque $\Gamma_{\text {em }}(10)$, and seven geometric inequalities (see Table II), $\Gamma_{\text {fixed }}$ is the value of torque imposed by the schedule of conditions; $t_{y i}^{\min }, g^{\mathrm{min}}, t_{y e}^{\min }$, and $R_{\mathrm{int}}^{\min }$ are respectively the minimum values of the thicknesses of the interior magnets, the airgap, the exterior magnets and the inner radius of our device; $g^{\max }$ is the maximal value of the airgap; $\lambda=\left[\lambda^{l}, \lambda^{u}\right]$ is a shape coefficient. 
TABLE III

RESULTS

\begin{tabular}{|c|c|c|c|c|}
\hline Parameters & Bounds & Unit & Min $\mathbf{V}_{\mathrm{a}}$ & Min $\mathbf{V}_{\mathrm{g}}$ \\
\hline$\theta_{\text {int }}$ & {$[0.001 ; 1] \pi / \mathrm{p}$} & $\mathrm{rad}$ & 38.15 & 48.65 \\
\hline$\theta_{\text {int }}$ & {$[0.001 ; 1] \pi / \mathrm{p}$} & $\mathrm{rad}$ & 25.75 & 37.80 \\
\hline$R_{1}$ & {$[10 ; 200]$} & $\mathrm{mm}$ & 26.785 & 22.015 \\
\hline$R_{2}$ & {$[10 ; 200]$} & $\mathrm{mm}$ & 29.785 & 25.015 \\
\hline$R_{3}$ & {$[10 ; 200]$} & $\mathrm{mm}$ & 31.785 & 27.015 \\
\hline$R_{4}$ & {$[10 ; 200]$} & $\mathrm{mm}$ & 34.785 & 30.015 \\
\hline$L$ & {$[50 ; 500]$} & $\mathrm{mm}$ & 50.22 & 50.025 \\
\hline$p$ & {$[2,7]$} & - & 7 & 4 \\
\hline$\sigma_{\mathrm{ai}}$ & {$[1,2]$} & - & 2 & 2 \\
\hline$\sigma_{\mathrm{ae}}$ & {$[1,2]$} & - & 2 & 2 \\
\hline$\sigma_{\mathrm{vi}}$ & {$[1,2]$} & - & 1 & 2 \\
\hline$\sigma_{\mathrm{ye}}$ & {$[1,2]$} & - & 1 & 2 \\
\hline$t_{\mathrm{v}}{ }^{3}$ & & $\mathrm{~mm}$ & 1.177 & 1.724 \\
\hline$t_{\mathrm{y}}{ }^{3}$ & & $\mathrm{~mm}$ & 0.867 & 1.448 \\
\hline$V_{a}$ & & $\mathrm{~cm}^{3}$ & $\mathbf{1 8 . 3 3}$ & 20.95 \\
\hline$V_{g}$ & & $\mathrm{~cm}^{3}$ & 97.08 & $\mathbf{9 0 . 8 7}$ \\
\hline$\Gamma_{e m}$ & & $\mathrm{~N} . \mathrm{m}$ & 9.806 & 9.809 \\
\hline CPU Time & & hours & 161 & 117 \\
\hline Iterations & & - & 2210055 & 2035197 \\
\hline & & & & \\
\hline
\end{tabular}

In front of the complexity and the strong nonlinearity of many expressions (criteria, torque, thicknesses of yoke), the use of interval arithmetic is not easy. The returned bounds are not efficient enough. Hence, to improve the convergence of IBBA, we propose to intersect $\Gamma_{e m}$ with an undervaluation $\Gamma_{e m}^{\text {inf }}$ and an overvaluation of it $\Gamma_{\mathrm{em}}^{\mathrm{sup}}$. We use for $\Gamma_{\mathrm{em}}^{\mathrm{inf}}$

$$
\begin{aligned}
\Gamma_{e m}^{\inf }= & \frac{2.10^{7} p^{2} L\left(R_{4}^{p+1}-R_{3}^{p+1}\right)\left(R_{2}^{p+1}-R_{1}^{p+1}\right)}{\pi^{2}(p+1)^{2}\left(R_{4}^{2 p}-R_{1}^{2 p}\right)} \\
& \times J_{r}\left(\sigma_{m i}\right) \sin \left(p \theta_{\text {int }} / 2\right) J_{r}\left(\sigma_{m e}\right) \sin \left(p \theta_{\text {ext }} / 2\right) .
\end{aligned}
$$

This procedure replaces the constraint $C_{1}$. An example can explain it. If, during the process, our one-column input vector $\{\mathbf{X}\}$ is:

$$
\{\mathbf{X}\}^{T}=\left\{\begin{array}{c}
{[0.637,0.649] \times \pi / 5,[0.662,0.675] \times \pi / 5} \\
{[0.297,0.320],} \\
{[0.300,0.324],[0.373,0.397],[0.376,0.400],} \\
{[0.466,0.480],[5,5],[1,1],[2,2],[2,2],[2,2]}
\end{array}\right\} .
$$

Therefore, the obtained intervals of the torque without and with these limiting actions are, respectively:

$$
\begin{aligned}
\Gamma_{e m} & =[-55513,50604] \\
\Gamma_{e m} \bigcap\left[\min \left(\Gamma_{e m}^{\mathrm{inf}}\right), \max \left(\Gamma_{e m}^{\mathrm{sup}}\right)\right] & =[-6589,1064] .
\end{aligned}
$$

A same method has been applied to $t_{y}^{1}$ and $t_{y}^{3}$, [9].

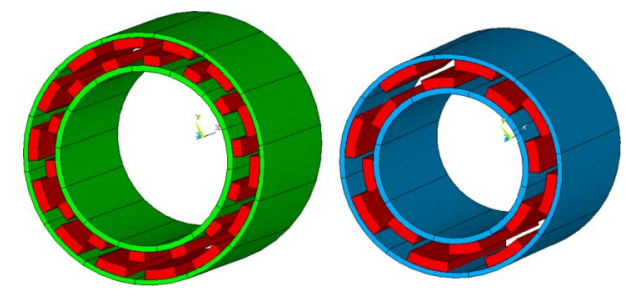

Fig. 2. Geometries of the two optimal solution (Min $\mathbf{V}_{\mathbf{a}}$ and $\operatorname{Min} \mathbf{V}_{\mathbf{g}}$ ).

\section{RESUlTS AND CONCLUSION}

To illustrate the use of this new IBBA algorithm, let us consider the following optimal design problem. It deals with the minimization of our two criteria $\left(\mathbf{V}_{\mathbf{a}}\right.$ and $\left.\mathbf{V}_{\mathbf{g}}\right)$. The equality constraint is true when the torque is equal to $10 \pm 0.2 \mathrm{~N} \cdot \mathrm{m}$. The magnets can be in $\mathrm{Sm} 2 \mathrm{Co} 17$ (if $\sigma_{m i}$ or $\sigma_{m e}=1$ ) or in $\mathrm{NdFeB}$ (if $\sigma_{m i}$ or $\sigma_{m e}=2$ ). The iron yokes can be in steel z15 (if $\sigma_{y i}$ or $\sigma_{y e}=1$ ) or in z30c13 (if $\sigma_{y i}$ or $\sigma_{y e}=2$ ). The initial bounds of our parameters and the obtained results are shown in Table III and on Fig. 2. First we can see that the value of torque is close to the allowed lower limit $(9.8 \mathrm{~N} \cdot \mathrm{m})$. We can also note that the obtained numbers of pole pairs are clearly different (4 and 7). Moreover the kinds of steel of yokes are different.

These results show the efficiency of our approach. Indeed without the dimensions, the integer and the categorical parameters describe a set of $116\left(7 \times 2^{4}\right)$ different geometries of magnetic couplings. The method is able to find the most efficient one and the result is guaranteed.

\section{REFERENCES}

[1] F. Messine, B. Nogarede, and J.-L. Lagouanelle, "Optimal design of electromechanical actuators: A new method based on global optimization," IEEE Trans. Magn., vol. 34, no. 1, pp. 299-308, Jan. 1998.

[2] E. Fitan, F. Messine, and B. Nogarede, "The electromagnetic actuator design problem: A general and rational approach," IEEE Trans. Magn., vol. 40, no. 3, pp. 1579-1590, Mar. 2004.

[3] F. Messine, "A deterministic global optimization algorithm for design problems," in Essays and Surveys in Global Optimization, C. Audet, P. Hansen, and G. Savard, Eds. Montreal, QC, Canada: GERAD, 2005 pp. 267-294.

[4] M. Couderc, C. Henaux, and B. Nogarede, "Analytical modelling of high speed electrical machines in view of their optimal design," presented at the IEEE 32nd Ann. Conf. Ind. Electron. (IECON), Paris, France, 2006.

[5] Z. Q. Zhu, D. Howe, B. Ekkehard Bolte, and A. Bemd, "Instantaneous magnetic field distribution in brushless permanent magnet $\mathrm{dc}$ motors, Part I: Open-circuit field," IEEE Trans. Magn., vol. 29, no. 1, pp. 124-134, Jan. 1993.

[6] R. E. Moore, Interval Analysis. Englewood Cliffs, NJ: Prentice-Hall, 1966.

[7] H. Ratschek and J. Rokne, New Computer Methods for Global Optimization. Chichester, U.K.: Ellis Horwood, 1988.

[8] F. Messine, "Deterministic global optimization using interval constraint propagation techniques," RAIRO Oper. Res., vol. 38, no. 4, pp. 277-294, 2004.

[9] J. Fontchastagner, "Résolution du problème inverse de conception d'actionneurs électromagnétiques par association de méthodes déterministes d'optimisation globale avec des modèles analytiques et numériques," Ph.D. dissertation, Elect. Eng. Dept., Univ. Toulouse, INPT, Toulouse, France, 2007. 